\title{
Geostatistical Analysis of the Colorada and Quimichule Canyons Located in Popocatépetl Volcano (Mexico) for the Prevention of Natural Disasters
}

\author{
Rogelio Ramos-Aguilar1, Patricia Máximo-Romero', Blanca Susana Soto-Cruz², \\ Salvador Alcántara-Iniesta ${ }^{2}$, María de la Cruz Vázquez-García ${ }^{3}$ \\ ${ }^{1}$ Engineering School, Benemérita Universidad Autónoma de Puebla, Puebla, México \\ ${ }^{2}$ Research Center of Semiconductor Devices, Benemérita Universidad Autónoma de Puebla, Puebla, México \\ ${ }^{3}$ Bufete in Telecommunications and Systems Engineering, México City, México \\ Email: rogelio.ramos@correo.buap.mx,patricia.maximo@correo.buap.mx
}

Received 13 January 2016; accepted 22 May 2016; published 25 May 2016

Copyright (C) 2016 by authors and Scientific Research Publishing Inc.

This work is licensed under the Creative Commons Attribution International License (CC BY). http://creativecommons.org/licenses/by/4.0/

(c) (i) Open Access

\begin{abstract}
This paper aims to contribute to the prevention of natural disasters and generate a complement to other similar studies. The Popocatépetl volcano has showed significant and constant activity since 1994. The Colorada and Quimichule canyons are located within its geologic structure; due to their topographic features, ejected volcanic material and torrential rains in the past recent years, they put nearby communities at risk. This work presents a geostatistical analysis to obtain the gravity acceleration, slope by the distance-elevation relation, height-gravity and the fluid force on the canyons. The conversion of UTM to geographical coordinates was made with the use of the program Traninv applying the ITRF2008 epoch 2010.0 Datum and the 14 Zone; the local gravity was calculated with the use of International Organization of Legal Metrology (OIML) and the statistical analysis was obtained with the use of the Geostatistical Environmental Assessment. The structural modeling was performed using Surfer, and the spending and force were calculated using hydrological models. The correlation analysis concluded that Quimichule has the greatest gravity and that it would transport lahars faster. Mapping, geomorphological and statistical techniques and models were applied in accordance with the study to obtain the results presented here.
\end{abstract}

Keywords

Natural Disasters, Geostatistical, Force, Acceleration, Gravity

How to cite this paper: Ramos-Aguilar, R., Máximo-Romero, P., Soto-Cruz, B.S., Alcántara-Iniesta, S. and Vázquez-García, M. de la C. (2016) Geostatistical Analysis of the Colorada and Quimichule Canyons Located in Popocatépetl Volcano (Mexico) for the Prevention of Natural Disasters. International Journal of Geosciences, 7, 701-715. 


\section{Introduction}

The Popocatépetl volcano is located at $19^{\circ} 17^{\prime} \mathrm{N}$ latitude - $98^{\circ} 38^{\prime} \mathrm{W}$ longitude from the Greenwich Meridian. Its height is 5520 meters above sea level and it borders with the states of Puebla, Morelos and Mexico. In 1994, the Popocatépetl volcano began an important stage of activity presenting in 1994, an important stage of steam, ash and incandescent rock ejection, as well as seismic events. It has remained active presenting both high and low intensity periods ever since. Its constant activity, atypical torrential rains caused by climate change, existing melting glaciers and topography, favor landslides of mudflows of volcanic ash in the canyons such as Colorada and Quimichule. These are directly related to the gravity, slope, height and expense, which might put the surrounding communities at risk. Furthermore, the pressure changes generated by the activity within the magma chamber cause gravity deformations and variations on the surface of the volcano-which must be quantified periodically. A study named "Possible Mudflow in the possible mudflow on the east side of the Popocatépetl volcano" [1] was carried out after the eruption in 1994. This study built a profile of the El Aguardientero canyon; its slopes were calculated every $100 \mathrm{~m}$ of elevation, using topographic maps of the National Institute of Statistics and Geography (INEGI) at a 1:50.000 scale, determining that one of the areas with the highest accumulation of ash is San Pedro Benito Juárez.

This paper aims to contribute to the prevention of natural disasters caused by volcanoes, specifically through the development of geostatistical methodologies. This information will be useful to determine which prevention actions to take in the event of volcanic activity created by thaw when a major volcanic activity exists. Currently, studies continue to be conducted in order to calculate other geomorphologic variables.

Studies on canyon stability have been carried out applying geotechnical methods and finite elements to determine tensions, deformations and shear strength [2]. These methods are based on mathematical models that provide an approach to the solution of the problem; however, they do not take into account geodetic methods to determine the slope and gravity. Geodetic approximate methods provide more realistic results, as the data introduced to the computer programs are obtained from readings on topographic maps with a good approach.

The location of this volcano and its activity in recent years represent a risk structure to the nearby communities (Figure 1).

Regarding the work in this paper, there is no information on similar studies or research in the area of Popocatépetl. Still, it is important to know the possible scenarios that would arise in the case of a major eruption.

\section{Materials and Methods}

The UTM coordinates of the canyons' profile were obtained through digital topographic maps of the volcano readings and are available to users on the website of the NATIONAL INSTITUTE OF STATISTICS AND GEOGRAPHY. Using these maps, we defined the points where the acceleration of gravity, distance-lift slope and gravity-height would be calculated. The UTM coordinates were converted to geographic coordinates using the Traninv program applying the ITRF2008 Datum and Zone 14. Currently, all the mapping and digital products produced by INEGI, subsequent to the date of December 2010 is based on the new Datum ITRF2008, also

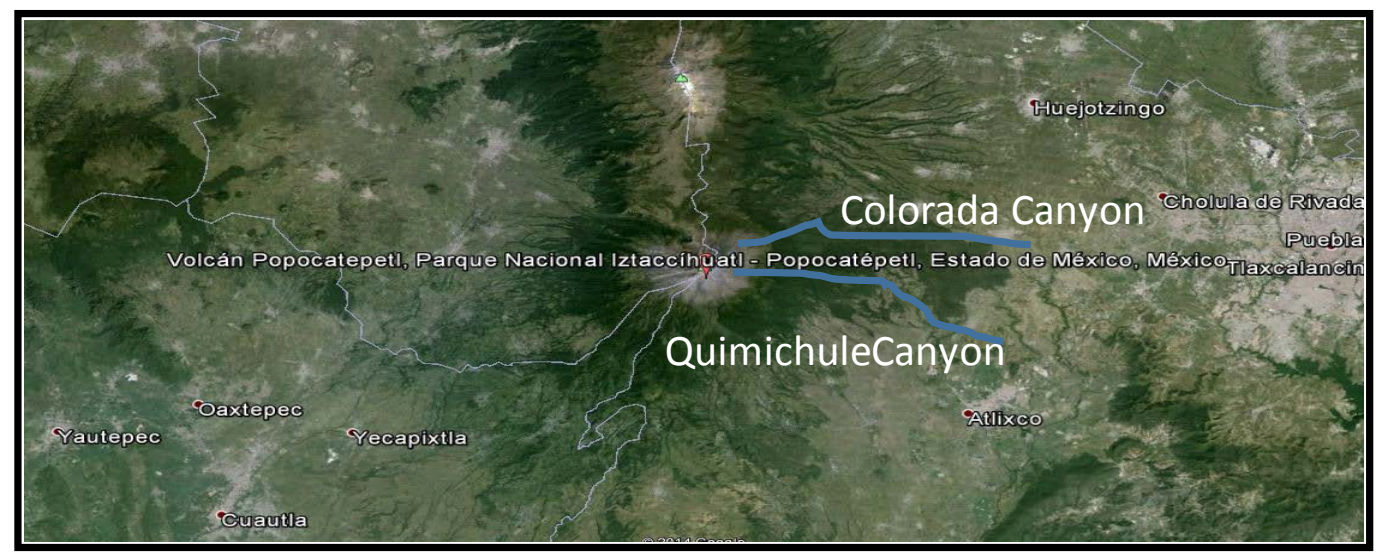

Figure 1. Topography of the Popocatépetl volcano in the National Izta-Popo Park, Mexico. 
releasing at the same time a software (Traninv) based on the mathematical algorithm for the transformation or conversion of coordinate ITRF92 to ITRF2008, which seeks a smooth transition to a new reference system. The acceleration of local gravity was calculated using the program on the National Metrology Center (Mexico) website, based on the OIML-N. 127 of 1992 newsletter.

The statistical analysis was carried out using the Geostatistical Environmental Assessment, and the structural modeling using the Surfer program. In addition, hydrological models were used to calculate the expense and force of a liquid.

Later, relations between gravity-height and gravity-slope were obtained, as well as their corresponding correlation coefficients. The correlation between the three studied variables is important because it defines the behavior of flows over the canyons, giving an idea location of the area of greatest risk in those geological structures.

\subsection{Converting the Coordinates of Recorded Data}

For each canyon data, the data was obtained from the E14B42 topographic map, getting the UTM coordinates and then transforming them to geographical coordinates using the Traninv program where $\mathrm{X}=\mathrm{W}$ and $\mathrm{Y}=\mathrm{N}$ (Figure 2).

The data used for the processing each $500 \mathrm{~m}$ in length for both canyons is shown on Table 1 and Table 2 .

\subsection{Development for the Calculating of the Acceleration of Local Gravity}

The acceleration of gravity is the act of universal attraction that propels bodies to the center of the Earth; it is a force that determines the weight of bodies [2]. The acceleration of gravity is by the letter $g$ and it is defined as the constant increase of velocity by time unit on a body in free fall, it is inversely proportional to the body massin kilograms $(\mathrm{kg}) g=F / m$. In order to calculate the local gravity of each canyon, the program recommended by the International Committee of Legal Metrology (OIML) was applied (Figure 3).

The Equation (1) was used to confirm the results of gravity in different points of the canyon every 500 meters of distance, which can be calculated accurately in the $0.001 \%=100 \mathrm{ppm}$ order. Introduced data includes: altitude (m) and North latitude $\left({ }^{\circ}\right)$. This program calculates the acceleration of local gravity by applying the Equation (1) (Table 3 and Table 4).

$$
g l=\left[g e *\left(1+f^{\prime} * \operatorname{sen}^{2} \phi\right)-\left(F_{4} * \operatorname{sen}^{2} 2 \phi\right)\right]-(D g * h) \quad \text { Model }
$$

where:

$g l=$ acceleration of local gravity $\left(\mathrm{m} / \mathrm{s}^{2}\right)$.

$g e=9.7803185 \mathrm{~m} / \mathrm{s}^{2}$, acceleration of gravity in the Equator $(\varphi=0)$.

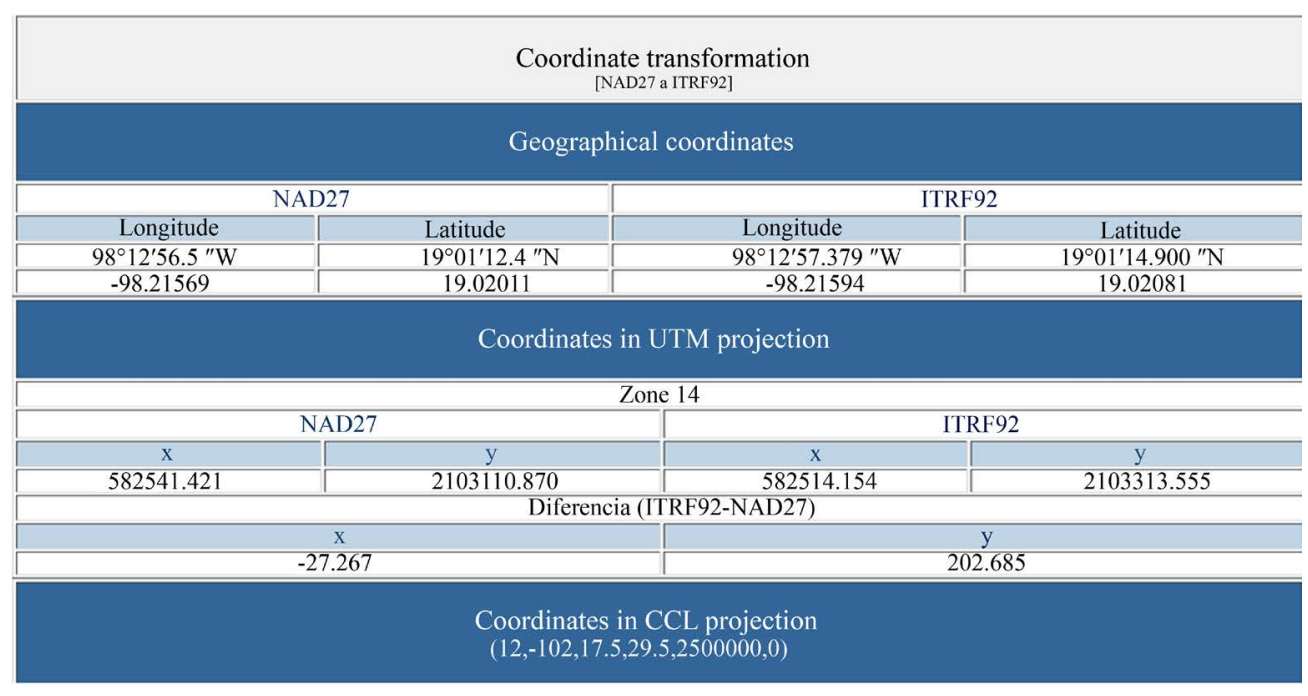

Figure 2. Transformation model of the UTM coordinates to geographical coordinates for all the readings of the canyons. 
Table 1. The Colorada canyon has a length of $6.5 \mathrm{~km}$, thus 13 readings were carried out $(\mathrm{Z}=$ height, $\mathrm{N}=$ latitude, $\mathrm{W}=$ length, in meters).

\begin{tabular}{|c|c|c|c|c|c|}
\hline \multicolumn{6}{|c|}{ Data } \\
\hline Point & Latitude & Longitude & $\mathbf{Z}$ & $\mathbf{W}$ & $\mathbf{N}$ \\
\hline 1 & $19^{\circ} 1^{\circ} 46.451^{\circ}$ & $98^{\circ} 37^{\circ} 23.916^{\circ}$ & 4880 & 539640.388 & 2104015.758 \\
\hline 2 & $19^{\circ} 1^{\circ} 39.899^{\circ}$ & $98^{\circ} 37^{\circ} 07.464^{\circ}$ & 4580 & 540121.747 & 2103815.432 \\
\hline 3 & $19^{\circ} 1^{\circ} 33.707^{\circ}$ & $98^{\circ} 36^{\circ} 51.840^{\circ}$ & 4280 & 540578.888 & 2103626.111 \\
\hline 4 & $19^{\circ} 1^{\circ} 26.616^{\circ}$ & $98^{\circ} 36^{\circ} 36.287^{\circ}$ & 4040 & 540034.0525 & 2103409.175 \\
\hline 5 & $19^{\circ} 1^{\circ} 21.071^{\circ}$ & $98^{\circ} 36^{\circ} 20.015^{\circ}$ & 3820 & 541510.084 & 2103239.814 \\
\hline 6 & $19^{\circ} 1^{\circ} 14.628^{\circ}$ & $98^{\circ} 36^{\circ} 03.888^{\circ}$ & 3580 & 541981.976 & 2103042.855 \\
\hline 7 & $19^{\circ} 1^{\circ} 08.400^{\circ}$ & $98^{\circ} 35^{\circ} 48.119^{\circ}$ & 3440 & 542443.398 & 2102852.492 \\
\hline 8 & $19^{\circ} 0^{\circ} 01.668^{\circ}$ & $98^{\circ} 35^{\circ} 31.236^{\circ}$ & 3280 & 542937.432 & 2102646.725 \\
\hline 9 & $19^{\circ} 0^{\circ} 54.404^{\circ}$ & $98^{\circ} 35^{\circ} 15.396^{\circ}$ & 3120 & 543401.024 & 2102424.549 \\
\hline 10 & $19^{\circ} 0^{\circ} 49.14^{\circ}$ & $98^{\circ} 34^{\circ} 59.628^{\circ}$ & 3000 & 543862.377 & 2102263.849 \\
\hline 11 & $19^{\circ} 0^{\circ} 44.280^{\circ}$ & $98^{\circ} 34^{\circ} 47.460^{\circ}$ & 2960 & 544218.462 & 2102115.326 \\
\hline
\end{tabular}

Table 2. The Quimichule canyon has a length of $6.5 \mathrm{~km}$, thus 13 readings were carried out $(\mathrm{Z}=$ height, $\mathrm{N}=$ latitude, $\mathrm{W}=$ length, in meters).

\begin{tabular}{|c|c|c|c|c|c|}
\hline \multicolumn{6}{|c|}{ Data } \\
\hline Point & Latitude & Longitude & $\mathbf{Z}$ & $\mathbf{W}$ & $\mathbf{N}$ \\
\hline 1 & $19^{\circ} 4^{\circ} 25.824^{\circ}$ & $98^{\circ} 36^{\circ} 11.16^{\circ}$ & 3400 & 541756.3216 & 2108918.483 \\
\hline 2 & $19^{\circ} 4^{\circ} 14.087^{\circ}$ & $98^{\circ} 36^{\circ} 21.995^{\circ}$ & 3500 & 541440.2879 & 2108557.144 \\
\hline 3 & $19^{\circ} 4^{\circ} 3.9^{\circ}$ & $98^{\circ} 36^{\circ} 35.244^{\circ}$ & 3587.872 & 541054.0718 & 2108243.504 \\
\hline 4 & $19^{\circ} 3^{\circ} 52.055^{\circ}$ & $98^{\circ} 36^{\circ} 46.835^{\circ}$ & 3651.994 & 540715.6962 & 2107878.453 \\
\hline 5 & $19^{\circ} 3^{\circ} 39.996^{\circ}$ & $98^{\circ} 36^{\circ} 55.512^{\circ}$ & 3738.675 & 540463.5108 & 2107507.299 \\
\hline 6 & $19^{\circ} 3^{\circ} 24.66^{\circ}$ & $98^{\circ} 37^{\circ} 0.804^{\circ}$ & 3820.266 & 540309.7976 & 2107035.544 \\
\hline 7 & $19^{\circ} 3^{\circ} 11.087^{\circ}$ & $98^{\circ} 37^{\circ} 8.4^{\circ}$ & 3881.08 & 540088.629 & 2106617.608 \\
\hline 8 & $19^{\circ} 2^{\circ} 56.759^{\circ}$ & $98^{\circ} 37^{\circ} 15.348^{\circ}$ & 3978.361 & 539885.7634 & 2106177.386 \\
\hline 9 & $19^{\circ} 2^{\circ} 41.64^{\circ}$ & $98^{\circ} 37^{\circ} 17.436^{\circ}$ & 4149.287 & 539826.3458 & 2105712.186 \\
\hline 10 & $19^{\circ} 2^{\circ} 27.42^{\circ}$ & $98^{\circ} 37^{\circ} 14.52^{\circ}$ & 4291.082 & 539912.5866 & 2105275.253 \\
\hline 11 & $19^{\circ} 2^{\circ} 11.327^{\circ}$ & $98^{\circ} 37^{\circ} 14.808^{\circ}$ & 4491.679 & 539904.7367 & 2104780.647 \\
\hline 12 & $19^{\circ} 1^{\circ} 56.567^{\circ}$ & $98^{\circ} 37^{\circ} 21.576^{\circ}$ & 4760.342 & 539708.4806 & 2104326.534 \\
\hline 13 & $19^{\circ} 1^{\circ} 46.74^{\circ}$ & $98^{\circ} 37^{\circ} 22.835^{\circ}$ & 4962.988 & 539672.0045 & 2104024.676 \\
\hline
\end{tabular}

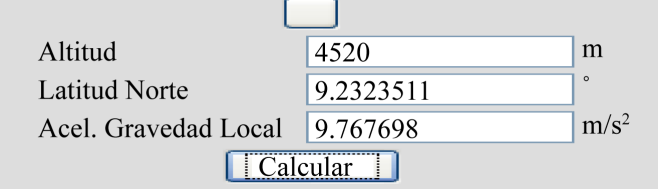

Figure 3. Example of the calculation of the acceleration of local gravity. 


\begin{tabular}{|c|c|c|c|c|c|c|c|c|c|c|c|c|}
\hline \multicolumn{13}{|c|}{ CALCULATION OF PARTIAL ACCELERATION OF GRAVITY } \\
\hline \multirow{2}{*}{ Pnt. } & \multicolumn{2}{|c|}{ COORDINATES } & \multirow{2}{*}{$\begin{array}{l}\text { Altitude } \\
\text { (Z) }\end{array}$} & \multicolumn{3}{|c|}{ Latitude $^{\circ}$} & \multirow{2}{*}{ (Decimal) } & \multirow{2}{*}{$\operatorname{sen}^{2}$} & \multirow{2}{*}{$\operatorname{Sen}^{2} 2$} & \multirow{2}{*}{$\begin{array}{c}\text { Local } \\
\text { gravity } \\
\text { acceleration } \\
(g l)\end{array}$} & \multirow{2}{*}{\multicolumn{2}{|c|}{$\begin{array}{l}\text { Constants to calculate the } \\
\text { acceleration of gravity of a point } \\
\text { with latitude different Ecuador }(g e)\end{array}$}} \\
\hline & $\mathbf{w}$ & $\mathbf{N}$ & & 。 & ' & " & & & & & & \\
\hline 1 & 539640.388 & 2104015.758 & 4880 & 19 & 01 & 46.451 & 19.02956972 & 0.10631257 & 0.380041 & 9.770750 & \multicolumn{2}{|c|}{$g e=9.7803185 \mathrm{~m} / \mathrm{s}^{2}$} \\
\hline 2 & 540121.747 & 2103815.432 & 4580 & 19 & 01 & 39.899 & 19.02774972 & 0.10629299 & 0.379979 & 9.771675 & \multicolumn{2}{|l|}{$f^{\prime} 0.0053024$} \\
\hline 3 & 540578.888 & 2103626.111 & 4280 & 19 & 01 & 33.707 & 19.02602972 & 0.10627448 & 0.379921 & 9.772600 & \multicolumn{2}{|l|}{$f_{4}=0.0000059$} \\
\hline 4 & 540034.0525 & 2103409.175 & 4040 & 19 & 01 & 26.616 & 19.02406 & 0.10625329 & 0.379854 & 9.773339 & \multicolumn{2}{|c|}{ Dg 0.000003086} \\
\hline 5 & 541510.084 & 2103239.814 & 3820 & 19 & 01 & 21.071 & 19.02251972 & 0.10623673 & 0.379802 & 9.774017 & & \\
\hline 6 & 541981.976 & 2103042.855 & 3580 & 19 & 01 & 14.628 & 19.02073 & 0.10621748 & 0.379741 & 9.774757 & \multicolumn{2}{|l|}{$h=$ (altitude) } \\
\hline 7 & 542443.398 & 2102852.492 & 3440 & 19 & 01 & 8.400 & 19.019 & 0.10619887 & 0.379683 & 9.775188 & \multicolumn{2}{|l|}{ f $\quad X X^{\prime \prime}$} \\
\hline 8 & 542937.432 & 2102646.725 & 3280 & 19 & 01 & 1.668 & 19.01713 & 0.10617876 & 0.379619 & 9.775681 & \multicolumn{2}{|l|}{ (latitude) } \\
\hline 9 & 543401.024 & 2102424.549 & 3120 & 19 & 00 & 54.404 & 19.01511222 & 0.10615706 & 0.379551 & 9.776173 & \multirow{2}{*}{\multicolumn{2}{|c|}{ Local Gravity Acceleration ( $g l)$}} \\
\hline 10 & 543862.377 & 2102263.849 & 3000 & 19 & 00 & 49.140 & 19.01365 & 0.10614134 & 0.379501 & 9.776543 & & \\
\hline \multirow[t]{5}{*}{11} & 544218.462 & 2102115.326 & 2960 & 19 & 00 & 44.280 & 19.0123 & 0.10612683 & 0.379456 & 9.776666 & \multirow{2}{*}{\multicolumn{2}{|c|}{$\begin{array}{l}g l= \\
{\left[g e^{*}\left(1+\left(f^{\prime *} \operatorname{sen}^{2} j\right)-\left(f_{4}^{*} \operatorname{sen}^{2} 2 j\right)\right)\right]} \\
-(D g * h)\end{array}$}} \\
\hline & & & & & & & & & & & & \\
\hline & & & & & & & & & & & \multicolumn{2}{|l|}{$\sum g l=107.517389928$} \\
\hline & & & & & & & & & & & \multicolumn{2}{|c|}{ Average Gravity Canyon: } \\
\hline & & & & & & & & & & & \multicolumn{2}{|l|}{9.774308} \\
\hline
\end{tabular}

Table 4. Quimichule canyon. Gravity was calculated every 500 meters resulting an average of $9.776018 \mathrm{~m} / \mathrm{s}^{2}$.

\begin{tabular}{|c|c|c|c|c|c|c|c|c|c|c|c|c|}
\hline \multicolumn{13}{|c|}{ CALCULATION OF PARTIAL ACCELERATION OF GRAVITY } \\
\hline \multirow{2}{*}{ Pnt. } & \multicolumn{2}{|c|}{ COORDINATES } & \multirow{2}{*}{$\begin{array}{l}\text { Altitude } \\
\text { (Z) }\end{array}$} & \multicolumn{3}{|c|}{ Latitude $^{\circ}$} & \multirow{2}{*}{ (Decimal) } & \multirow{2}{*}{$\operatorname{sen}^{2}$} & \multirow{2}{*}{$\operatorname{Sen}^{2} 2$} & \multirow{2}{*}{$\begin{array}{c}\text { Local gravity } \\
\text { acceleration } \\
(g l)\end{array}$} & \multirow{2}{*}{\multicolumn{2}{|c|}{$\begin{array}{l}\text { Constants to calculate the } \\
\text { acceleration of gravity of a point } \\
\text { with latitude different Ecuador (ge) }\end{array}$}} \\
\hline & $\mathbf{W}$ & $\mathbf{N}$ & & 。 & ' & $"$ & & & & & & \\
\hline 1 & 541756.3216 & 2108918.483 & 3400 & 19 & 4 & 25.824 & 19.07384 & 0.10678937 & 0.381542 & 9.775342 & \multicolumn{2}{|c|}{$g e=9.7803185 \mathrm{~m} / \mathrm{s}^{2}$} \\
\hline 2 & 541440.2879 & 2108557.144 & 3500 & 19 & 4 & 14.087 & 19.07057972 & 0.10675422 & 0.381431 & 9.775032 & \multicolumn{2}{|l|}{$f^{\prime} 0.0053024$} \\
\hline 3 & 541054.0718 & 2108243.504 & 3587.872 & 19 & 4 & 3.900 & 19.06775 & 0.10672372 & 0.381335 & 9.774759 & \multicolumn{2}{|l|}{$f_{4}=0.0000059$} \\
\hline 4 & 540715.6962 & 2107878.453 & 3651.994 & 19 & 3 & 52.055 & 19.06445972 & 0.10668826 & 0.381224 & 9.774559 & \multicolumn{2}{|l|}{ Dg 0.000003086} \\
\hline 5 & 540463.5108 & 2107507.299 & 3738.675 & 19 & 3 & 39.990 & 19.06110833 & 0.10665215 & 0.38111 & 9.774290 & & \\
\hline 6 & 540309.7976 & 2107035.544 & 3820.266 & 19 & 3 & 24.660 & 19.05685 & 0.10660627 & 0.380965 & 9.774036 & \multicolumn{2}{|l|}{$h=$ (altitude) } \\
\hline 7 & 540088.629 & 2106617.608 & 3881.08 & 19 & 3 & 11.087 & 19.05307972 & 0.10656566 & 0.380838 & 9.773846 & \multicolumn{2}{|l|}{ f $\quad X^{\prime \prime}$} \\
\hline 8 & 539885.7634 & 2106177.386 & 3978.361 & 19 & 2 & 56.759 & 19.04909972 & 0.10652279 & 0.380703 & 9.773543 & \multicolumn{2}{|l|}{ (latitude) } \\
\hline 9 & 539826.3458 & 2105712.186 & 4149.287 & 19 & 2 & 41.640 & 19.0449 & 0.10647757 & 0.38056 & 9.773014 & \multirow{2}{*}{\multicolumn{2}{|c|}{ Local Gravity Acceleration ( $g l$ ) }} \\
\hline 10 & 539912.5866 & 2105275.253 & 4291.082 & 19 & 2 & 27.420 & 19.04095 & 0.10643505 & 0.380427 & 9.772574 & & \\
\hline 11 & 539904.7367 & 2104780.647 & 4491.679 & 19 & 2 & 11.327 & 19.03647972 & 0.10638693 & 0.380275 & 9.771952 & \multirow{2}{*}{\multicolumn{2}{|c|}{$\begin{array}{l}g l= \\
{\left[g e^{*}\left(1+\left(f^{\prime} * \operatorname{sen}^{2} j\right)-\left(f_{4}^{*} \operatorname{sen}^{2} 2 j\right)\right)\right]} \\
-(D g * h)\end{array}$}} \\
\hline 12 & 539708.4806 & 2104326.534 & 4760.342 & 19 & 1 & 56.567 & 19.03237972 & 0.10634281 & 0.380136 & 9.771121 & & \\
\hline \multirow[t]{3}{*}{13} & 539672.0045 & 2104024.676 & 4962.988 & 19 & 1 & 46.740 & 19.02965 & 0.10631343 & 0.380044 & 9.770494 & $\sum g l=127.054561911$ & $\begin{array}{c}\text { Data } \\
13\end{array}$ \\
\hline & & & & & & & & & & & \multicolumn{2}{|c|}{ Average Gravity Canyon: } \\
\hline & & & & & & & & & & & \multicolumn{2}{|l|}{9.773427839} \\
\hline
\end{tabular}


$f^{\prime}=0.0053024$ (gravitational collapse)

$\phi=$ Latitude, in degrees, minutes, seconds $\left(00^{\circ} 00^{\prime} 00^{\prime \prime}\right)$

$h=$ Height above mean sea level (m)

$F_{4}=0.0000059$

$D g=0.000003086$

\subsection{Calculating the Slope}

The slope is the existing relation between the elevation and horizontal distance on a plane, which is equal to the tangent of the angle that forms the line to measure with the $\mathrm{X}$ axis. See Equation (2)

$$
m=\frac{y_{2}-y_{1}}{x_{2}-x_{1}} \quad \text { Model }
$$

Using the contour lines on the topographic map and applying the interpolation method, the slope of the canyons was determined taking readings every 500 meters along the channel. The arithmetic average was also calculated for the points in each canyon [3] (Table 5 and Table 6).

\section{Results}

\subsection{Geostatistical Analysis Using the Geoeas Program}

Through the use of the Geoeas program, which creates weighted normal probability graphics of the forcing variables (gravity and height), the statistical behavior of dispersion was obtained with the linear regression model of the matrix of the analyzed variables, which coefficient for each processed canyon is close to -1 (Figure 4).

Table 5. Colorada canyon.

\begin{tabular}{|c|c|c|c|c|c|c|}
\hline \multicolumn{7}{|c|}{ Slope of the Canyon } \\
\hline \multirow{2}{*}{ Pnt. } & \multicolumn{2}{|c|}{ COORDINATES } & \multirow{2}{*}{ Altitude } & \multirow{2}{*}{ Distance } & \multirow{2}{*}{$m=\frac{N_{2}-N_{1}}{W_{2}-W_{1}}$} & \\
\hline & $\mathbf{W}$ & $\mathbf{N}$ & & & & \\
\hline 1 & 539640.388 & 2104015.758 & 4880 & & & \multirow{3}{*}{$\begin{array}{c}\sum_{-3.840000000} m_{1}+m_{2}= \\
\end{array}$} \\
\hline 2 & 540121.747 & 2103815.432 & 4580 & 500 & -0.60000 & \\
\hline 3 & 540578.888 & 2103626.111 & 4280 & 500 & -0.60000 & \\
\hline 4 & 540034.0525 & 2103409.175 & 4040 & 500 & -0.48000 & \multirow[b]{2}{*}{ Average slope } \\
\hline 5 & 541510.084 & 2103239.814 & 3820 & 500 & -0.44000 & \\
\hline 6 & 541981.976 & 2103042.855 & 3580 & 500 & -0.48000 & \multirow[b]{2}{*}{-0.349090909} \\
\hline 7 & 542443.398 & 2102852.492 & 3440 & 500 & -0.28000 & \\
\hline 8 & 5422937.432 & 2102646.725 & 3280 & 500 & -0.32000 & High point \\
\hline 9 & 543401.024 & 2102424.549 & 3120 & 500 & -0.32000 & 4880 \\
\hline 10 & 543862.377 & 2102263.849 & 3000 & 500 & -0.24000 & Low point \\
\hline \multirow[t]{5}{*}{11} & 544218.462 & 2102115.326 & 2960 & 500 & -0.08000 & 2960 \\
\hline & & & & & & Distance \\
\hline & & & & & & 5000 \\
\hline & & & & & & $\begin{array}{l}\text { Slope between } \\
\text { points }\end{array}$ \\
\hline & & & & & & -0.384000000 \\
\hline
\end{tabular}


Table 6. Quimichule canyon.

\begin{tabular}{|c|c|c|c|c|c|c|}
\hline \multicolumn{7}{|c|}{ Slope of the canyon } \\
\hline \multirow{2}{*}{ Pnt. } & \multicolumn{2}{|c|}{ COORDINATES } & \multirow{2}{*}{ Altitude } & \multirow{2}{*}{ Distance } & \multirow{2}{*}{$m=\frac{N_{2}-N_{1}}{W_{2}-W_{1}}$} & \\
\hline & $\mathbf{w}$ & $\mathbf{N}$ & & & & \\
\hline 1 & 541756.3216 & 2108918.483 & 3400 & & & \multirow{2}{*}{$\begin{array}{c}\sum m_{1}+m_{2}= \\
3.125976000\end{array}$} \\
\hline 2 & 541440.2879 & 2108557.144 & 3500 & 500 & 0.20000 & \\
\hline 3 & 541054.0718 & 2108243.504 & 3587.872 & 500 & 0.17574 & \\
\hline 4 & 540715.6962 & 2107878.453 & 3651.994 & 500 & 0.12824 & \multirow[b]{2}{*}{ Average slope } \\
\hline 5 & 540463.5108 & 2107507.299 & 3738.675 & 500 & 0.17336 & \\
\hline 6 & 540309.7976 & 2107035.544 & 3820.266 & 500 & 0.16318 & \multirow[b]{2}{*}{0.240459692} \\
\hline 7 & 540088.629 & 2106617.608 & 3881.08 & 500 & 0.12163 & \\
\hline 8 & 539885.7634 & 2106177.386 & 3978.361 & 500 & 0.19456 & \\
\hline 9 & 539826.3458 & 2105712.186 & 4149.287 & 500 & 0.34185 & High point \\
\hline 10 & 539912.5866 & 2105275.253 & 4291.082 & 500 & 0.28359 & 4963 \\
\hline 11 & 539904.7367 & 2104780.647 & 4491.679 & 500 & 0.40119 & Low point \\
\hline 12 & 539708.4806 & 2104326.534 & 4760.342 & 500 & 0.53733 & 3400 \\
\hline \multirow[t]{4}{*}{13} & 539672.0045 & 2104024.676 & 4962.988 & 500 & 0.40529 & Distance \\
\hline & & & & & & 500 \\
\hline & & & & & & $\begin{array}{c}\text { Slope between } \\
\text { points }\end{array}$ \\
\hline & & & & & & -3.125976000 \\
\hline
\end{tabular}

With an overlay of the surfer model and the statistical representation deployed, the match between the processed and modeled data can be observed. There were also graphics created to show the behavior of each one of the considered variables [4].

Geostatistical analysis of the canyons was carried out, calculating the standard deviation for determining the arithmetic average of fluctuation of data from its mean or center point and the covariance, a joint dispersion measure of two statistics variables for each of the canyons [5]. The previous measures were used to obtain the correlation coefficient, which general result for each canyon is close to -1 . For statistics purposes, an analysis is presented in order to determine the covariance and correlation coefficient for each of the channels and to obtain their respective graphics, as shown in Table 7 and Table 8.

\subsection{Geostatistical Analysis Using SURFER}

The analysis of the statistical variables between height and gravity was obtained by applying the Surfer program [6]. By comparing the results of this analysis with the Geoeas graphics, it may be observed that the gravity process tends to approach the calculated value even as the altitude reduces (Table 9 and Table 10).

\subsection{Structural Modeling Applying SURFER}

The 2D and 3D structural modeling was made using Surfer, which was useful to identify the canyons of study and their vector behavior [7] (Figure 5 and Figure 6).

The variogram is a tool that allows analysis of the spatial behavior of a variable over a defined area [8].

In the case of the canyons, the variogram of the height was created against the local calculated gravity (Figure 7). 
Colorada Canyon

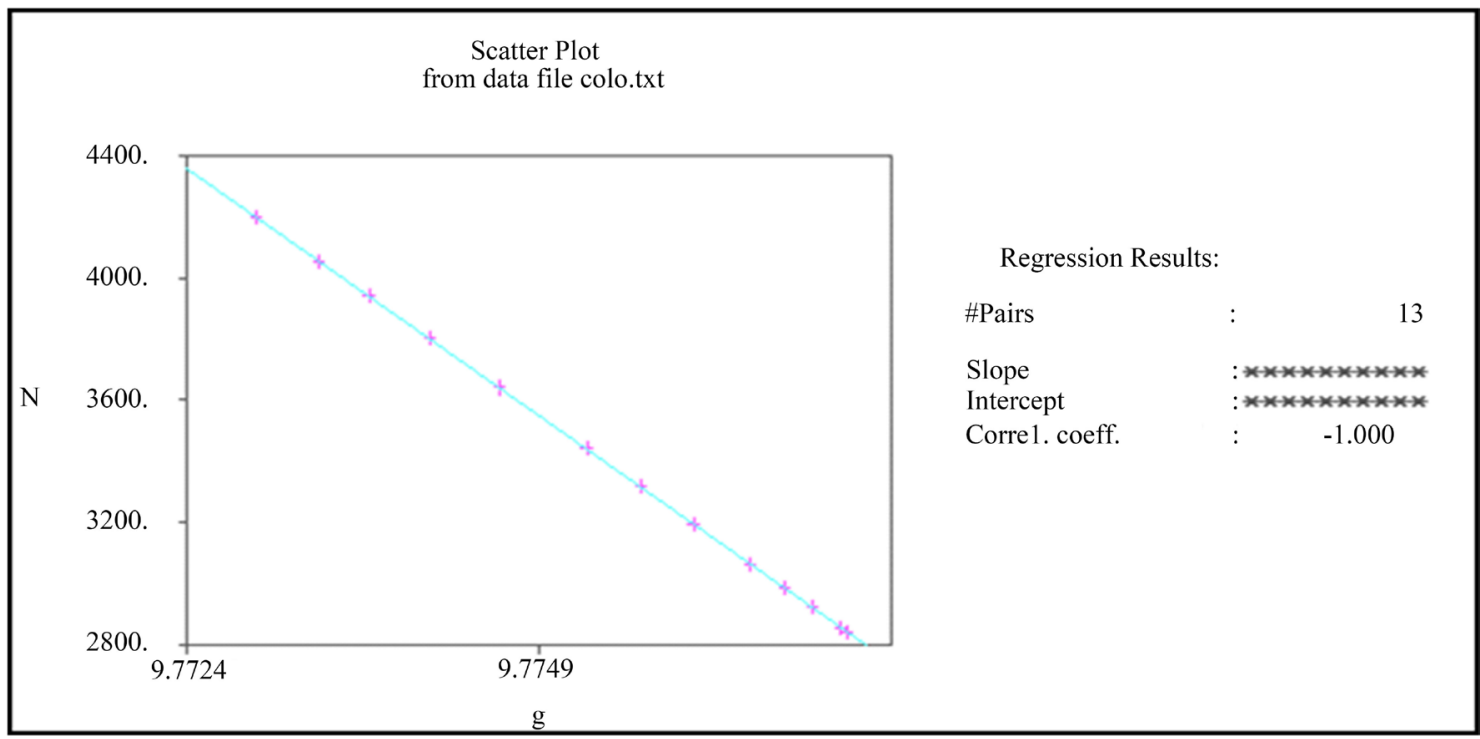

Quimichule Canyon

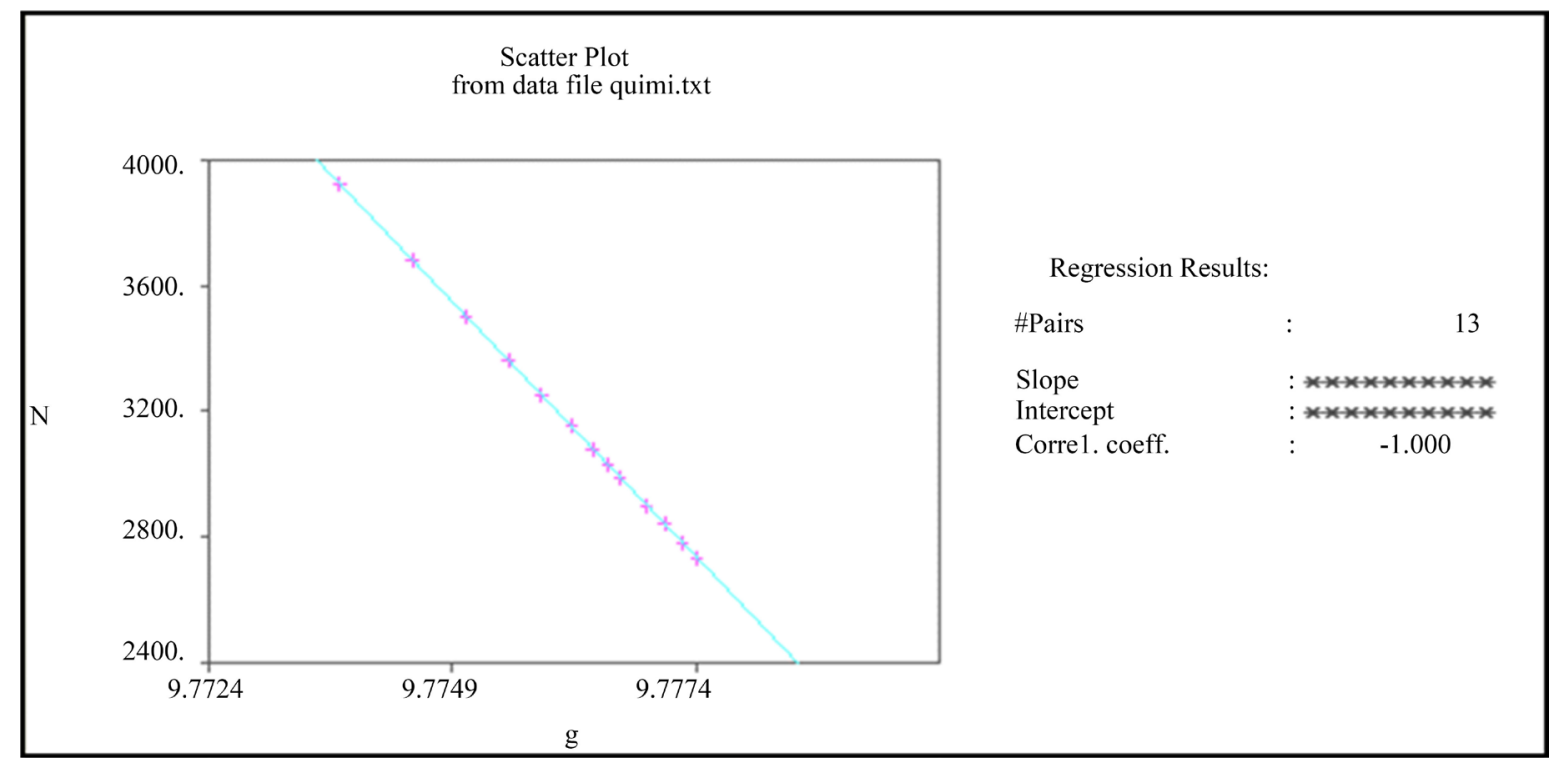

Figure 4. Correlation coefficient on the Colorada and Quimichule canyons.

\subsection{Calculating the Force of Water}

The force of water is the amount of thrust that this liquid exerts on the slope direction; the water density is 1 $\mathrm{gr} / \mathrm{cm}^{3}$ or $1000 \mathrm{Kg} / \mathrm{m}^{3}$. The calculations done using the mathematical model or Equation (3) showed that the force increases proportionally to the inclination of the slope.

$$
F_{1}=(d * \operatorname{cosm})(g l) \quad \text { Model }
$$

The water channel was subdivided in an equidistance of $500 \mathrm{~m}$ [9]; the calculations are shown in Table 11 and Table 12.

\subsection{Calculating the Waste}

The waste is the volume of a substance, which passes per unit of time. In this case, the waste of each of the can- 
Table 7. Colorada canyon.

\begin{tabular}{|c|c|c|c|c|c|c|c|c|c|}
\hline Pto. & Altitude (m) & & $\left(X_{i}-\bar{X}\right)^{2}$ & & $\begin{array}{c}\text { Local } \\
\text { gravity } \\
\text { acceleration } \\
\left(\mathrm{m} / \mathrm{s}^{2}\right)\end{array}$ & & $\left(Y_{i}-\bar{Y}\right)^{2}$ & & \\
\hline 1 & 4200 & 796.1538 & 633860.947 & & 9.772851 & -0.002458 & $6.0394 \mathrm{E}-06$ & & -1.956560877 \\
\hline 2 & 4055 & 651.1538 & 424001.331 & & 9.773300 & -0.002009 & $4.0373 \mathrm{E}-06$ & & -1.308367578 \\
\hline 3 & 3940 & 536.1538 & 287460.947 & & 9.773655 & -0.00165 & $2.736 \mathrm{E}-06$ & & -0.886845767 \\
\hline 4 & 3800 & 396.1538 & 156937.870 & & 9.774088 & -0.001221 & $1.4916 \mathrm{E}-06$ & & -0.48382825 \\
\hline 5 & 3640 & 236.1538 & 55768.639 & & 9.774582 & -0.000727 & $5.2901 \mathrm{E}-07$ & & -0.171762177 \\
\hline 6 & 3440 & 36.1538 & 1307.101 & & 9.775199 & -0.000110 & $1.2057 \mathrm{E}-08$ & & -0.003969814 \\
\hline 7 & 3315 & -88.8462 & 7893.639 & & 9.775585 & 0.000276 & $7.6147 \mathrm{E}-08$ & & -0.024516798 \\
\hline 8 & 3195 & -208.8462 & 43616.716 & & 9.775954 & 0.000645 & $4.1625 \mathrm{E}-07$ & & -0.134741456 \\
\hline 9 & 3065 & -338.8462 & 114816.716 & & 9.776355 & 0.001046 & $1.0945 \mathrm{E}-06$ & & -0.354493546 \\
\hline 10 & 2985 & -418.8462 & 175432.101 & & 9.776601 & 0.001292 & $1.6699 \mathrm{E}-06$ & & -0.541259438 \\
\hline 11 & 2920 & -483.8462 & 234107.101 & & 9.776801 & 0.001492 & $2.2259 \mathrm{E}-06$ & & -0.721869941 \\
\hline 12 & 2855 & -548.8462 & 301232.101 & & 9.777000 & 0.001691 & $2.8591 \mathrm{E}-06$ & & -0.928044829 \\
\hline 13 & 2840 & -563.8462 & 317922.485 & $\begin{array}{l}\text { Standard } \\
\text { deviation }\end{array}$ & 9.777046 & 0.001737 & $3.017 \mathrm{E}-06$ & $\begin{array}{l}\text { Standard } \\
\text { deviation }\end{array}$ & -0.979377112 \\
\hline & 3403.846154 & & 211873.669 & 460.2973698 & 9.775309 & & $2.0157 \mathrm{E}-06$ & 0.001419758 & -0.653510583 \\
\hline & & & \multicolumn{6}{|c|}{ Correlation coefficient } & Covariance \\
\hline
\end{tabular}

\begin{tabular}{|c|c|c|c|c|c|c|c|c|c|}
\hline Pto. & Altitude (m) & & $\left(X_{i}-\bar{X}\right)^{2}$ & & $\begin{array}{c}\text { Local } \\
\text { gravity } \\
\text { acceleration }\end{array}$ & & $\left(Y_{i}-\bar{Y}\right)^{2}$ & & \\
\hline 1 & 3920 & 750 & 562500.0000 & & 9.773706 & -0.002312 & $5.3435 \mathrm{E}-06$ & & -1.733698657 \\
\hline 2 & 3680 & 510 & 260100.0000 & & 9.774448 & -0.001570 & $2.4656 \mathrm{E}-06$ & & -0.800811723 \\
\hline 3 & 3500 & 330 & 108900.0000 & & 9.775002 & -0.001016 & $1.0323 \mathrm{E}-06$ & & -0.335282639 \\
\hline 4 & 3360 & 190 & 36100.0000 & & 9.775434 & -0.000584 & $3.414 \mathrm{E}-07$ & & -0.111015158 \\
\hline 5 & 3250 & 80 & 6400.0000 & & 9.775773 & -0.000245 & $6.0108 \mathrm{E}-08$ & & -0.019613542 \\
\hline 6 & 3150 & -20 & 400.0000 & & 9.776081 & 0.000063 & $3.9834 \mathrm{E}-09$ & & -0.00126228 \\
\hline 7 & 3080 & -90 & 8100.0000 & & 9.776297 & 0.000279 & $7.7683 \mathrm{E}-08$ & & -0.025084539 \\
\hline 8 & 3030 & -140 & 19600.0000 & & 9.776451 & 0.000433 & $1.8754 \mathrm{E}-07$ & & -0.060628639 \\
\hline 9 & 2990 & -180 & 32400.0000 & & 9.776574 & 0.000556 & $3.0938 \mathrm{E}-07$ & & -0.100119271 \\
\hline 10 & 2900 & -270 & 72900.0000 & & 9.776850 & 0.000832 & $6.9193 \mathrm{E}-07$ & & -0.224592674 \\
\hline 11 & 2840 & -330 & 108900.0000 & & 9.777033 & 0.001015 & $1.0307 \mathrm{E}-06$ & & -0.335033064 \\
\hline 12 & 2780 & -390 & 152100.0000 & & 9.777216 & 0.001198 & $1.4359 \mathrm{E}-06$ & & -0.467330943 \\
\hline 13 & 2730 & -440 & 193600.0000 & $\begin{array}{r}\text { Standard } \\
\text { deviation }\end{array}$ & 9.777369 & 0.001351 & $1.8247 \mathrm{E}-06$ & $\begin{array}{l}\text { Standard } \\
\text { deviation }\end{array}$ & -0.594357709 \\
\hline & 3170 & & 120153.8462 & 346.6321482 & 9.776018 & & $1.1388 \mathrm{E}-06$ & 0.001067156 & -0.369910064 \\
\hline & & & \multicolumn{6}{|c|}{ Correlation coefficient } & Covariance \\
\hline
\end{tabular}


Table 9. Colorada canyon.

\begin{tabular}{cccc}
\hline Univariate Statistics & X & & \\
\hline & 2840 & $\mathrm{Y}$ & $\mathrm{Z}$ \\
\hline Minimum: & 2985 & 9.772851392 & 9.772851392 \\
25\%-tile: & 3315 & 9.774087594 & 9.774087594 \\
Median: & 3800 & 9.775584855 & 9.775584855 \\
75\%-tile: & 4200 & 9.776601172 & 9.777045867172 \\
Maximum: & 3520 & 9.777045867 & 9.7749486295 \\
Midrange: & 1360 & 9.7749486295 & 0.0041944750000003 \\
Range: & 815 & 0.0041944750000003 & 0.0025135780000003 \\
Interquartile Range: & 395 & 0.00251357800000003 & 0.0012159950000008 \\
Median Abs. Deviation & 3403.8461538462 & 0.0012159950000008 & 9.7753089084615 \\
Mean: & 3382.7272727273 & 9.7753089084615 & 9.7753744137273 \\
Trim Mean (10\%): & 460.29736979376 & 9.7753744137273 & 0.0014197582068528 \\
Standard Deviation: & 211873.66863905 & $2.015713365926 \mathrm{E}-006$ & $2.015713365926 \mathrm{E}-006$ \\
Variance: & & & 0.00014523921649411 \\
Coef. of Variation & & & -0.34133729589633 \\
\hline
\end{tabular}

Table 10. Quimichule canyon.

\begin{tabular}{cccc}
\hline Univariate Statistics & & & \\
\hline & $\mathrm{X}$ & $\mathrm{Y}$ & $\mathrm{Z}$ \\
\hline Minimum: & 2730 & 9.773706207 & 9.773706207 \\
25\%-tile: & 2900 & 9.775433515 & 9.775433515 \\
Median: & 3080 & 9.776296522 & 9.776296522 \\
75\%-tile: & 3360 & 9.77684963 & 9.77684963 \\
Maximum: & 3920 & 9.777638618 & 9.777368618 \\
Midrange: & 3325 & 9.7755374125 & 9.7755374125 \\
Range: & 1190 & 0.0036624110000005 & 0.0014161149999996 \\
Interquartile Range: & 460 & 0.0014161149999996 & 0.00073653499999971 \\
Median Abs. Deviation & 240 & 0.00073653499999971 & 9.7760178050769 \\
Mean: & 3170 & 9.7760178050769 & 9.7761051491818 \\
Trim Mean (10\%): & 3141.8181818182 & 9.7761051491818 & 0.0010671560113215
\end{tabular}



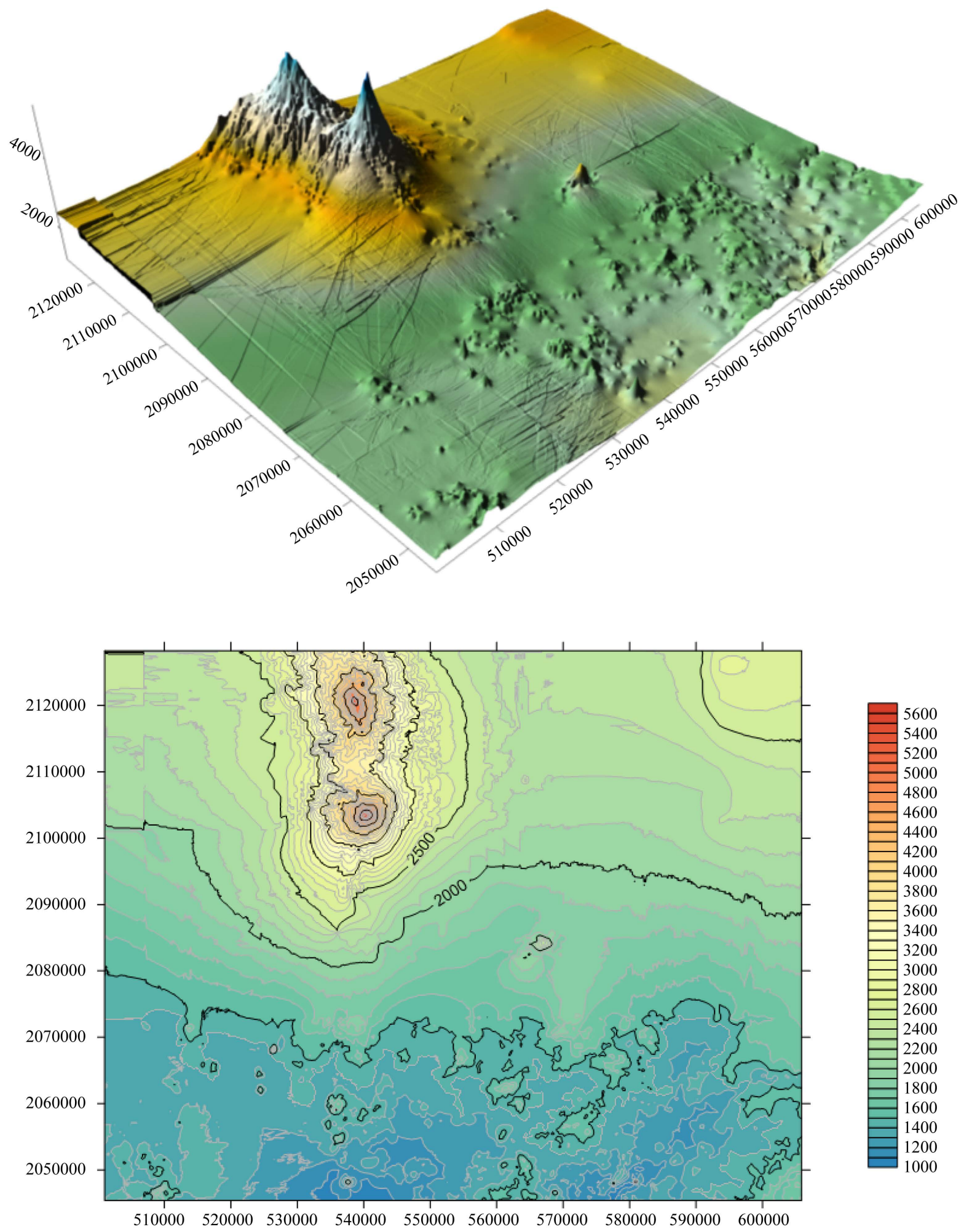

Figure 5. Identification of the canyons of study in the base map of the Popocatépetl volcano area.

yons was obtained by applying the equation of the rational method (4) (Table 13 and Table 14).

$$
Q=0.278 * k * i * A \quad \text { Model }
$$



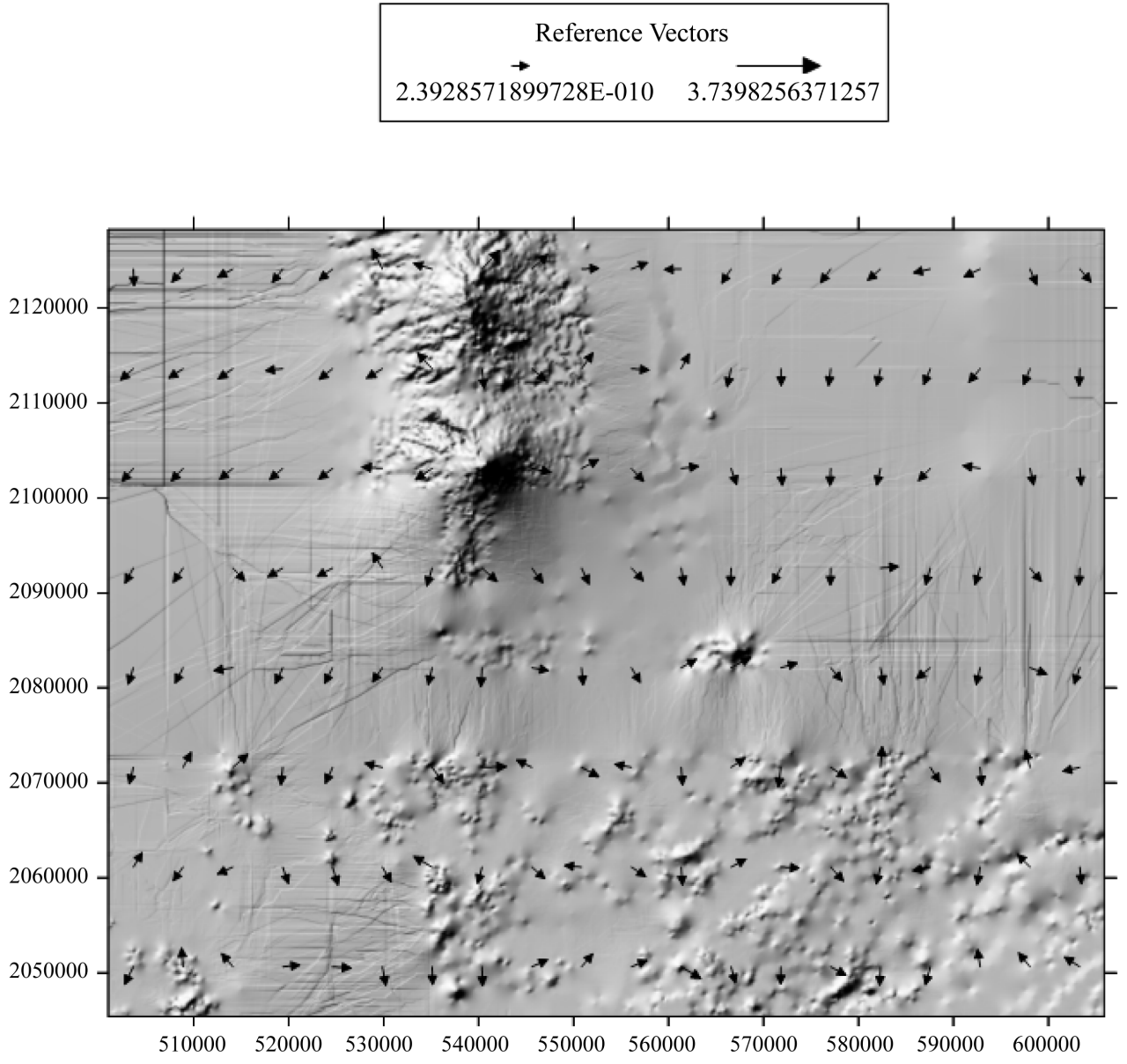

Figure 6. Vector direction of the canyons with respect of gravity.

Column D

Direction: 0.0 Tolerance: 90.0

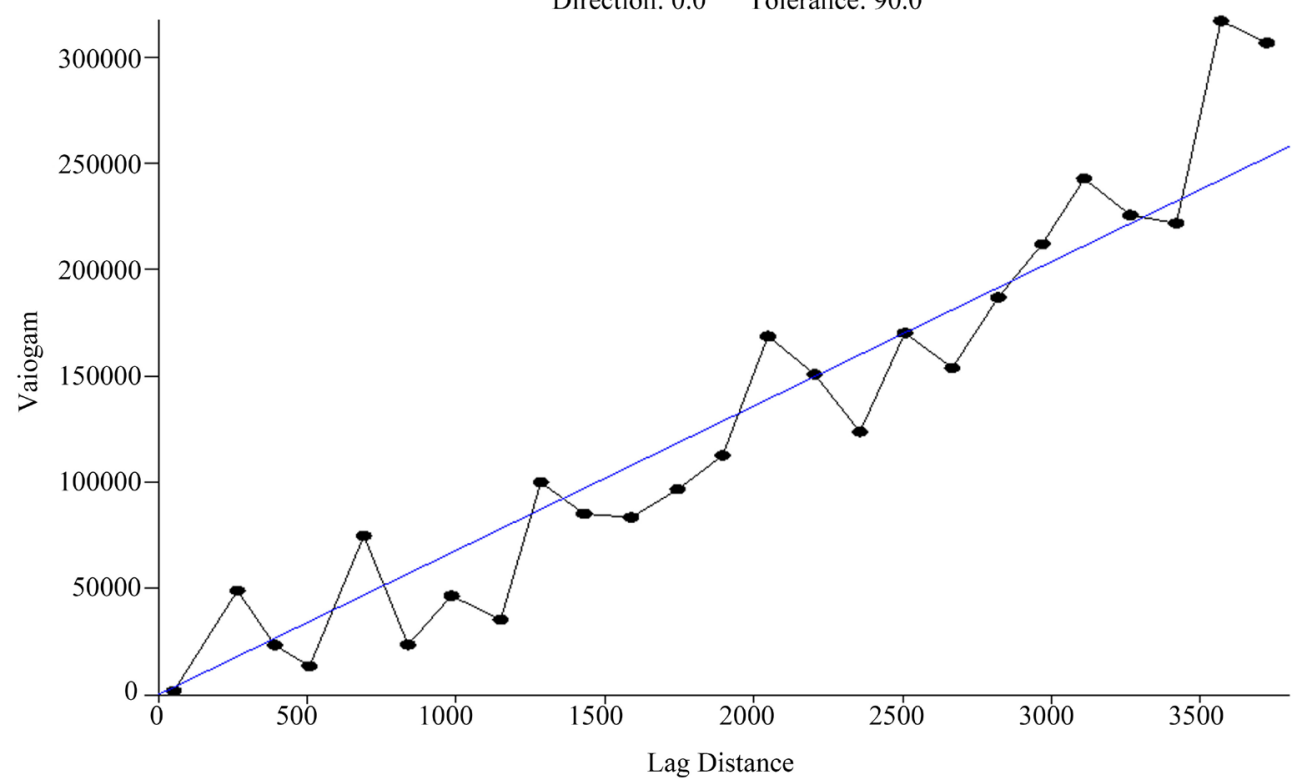

Figure 7. Variogram of the height with respect of gravity. 
Table 11. Colorada canyon.

\begin{tabular}{|c|c|c|c|c|c|c|c|}
\hline \multicolumn{8}{|c|}{ Strength calculation of water } \\
\hline Pto. & Altitude & $\begin{array}{l}\text { Density of } \\
\text { water }\end{array}$ & $m=\frac{N_{2}-N_{1}}{W_{2}-W_{1}}$ & $\begin{array}{l}\text { Cosine of the } \\
\text { slope (Cos m) }\end{array}$ & $\begin{array}{c}\text { Acceleration } \\
\text { gravity local (gl) }\end{array}$ & Partial forces & $F_{i}=\left(\mathrm{d}^{*} \operatorname{cosm}\right)(g l)$ \\
\hline \multicolumn{8}{|c|}{ Channel slope } \\
\hline 1 & 4200 & & & & & & $\Gamma E=117300306330$ \\
\hline 2 & 4055 & 1000 & -0.416168 & 0.999974 & 9.773300 & 97.730418 & $2 F_{i}-1173.00200300$ \\
\hline 3 & 3940 & 1000 & -0.414141 & 0.999974 & 9.773655 & 97.733995 & \\
\hline 4 & 3800 & 1000 & 0.398168 & 0.999976 & 9.774088 & 97.738516 & \multirow[b]{2}{*}{ Data } \\
\hline 5 & 3640 & 1000 & -0.114741 & 0.999998 & 9.774582 & 97.745620 & \\
\hline 6 & 3440 & 1000 & -0.417382 & 0.999973 & 9.775199 & 97.749397 & \multirow{3}{*}{12} \\
\hline 7 & 3315 & 1000 & -0.412557 & 0.999974 & 9.775585 & 97.753314 & \\
\hline 8 & 3195 & 1000 & -0.000042 & 1.000000 & 9.775954 & 97.759541 & \\
\hline 9 & 3065 & 1000 & 0.000046 & 1.000000 & 9.776355 & 97.763551 & \multirow{3}{*}{$F=\sum_{i=1}^{n} F_{i}$} \\
\hline 10 & 2985 & 1000 & -0.348323 & 0.999982 & 9.776601 & 97.764205 & \\
\hline 11 & 2920 & 1000 & -0.417100 & 0.999974 & 9.776801 & 97.765418 & \\
\hline 12 & 2855 & 1000 & 0.582900 & 0.999948 & 9.777000 & 97.764936 & \multirow{2}{*}{$97.75017194 \mathrm{~N}$} \\
\hline 13 & 2840 & 1000 & 1.582900 & 0.999618 & 9.777046 & 97.733150 & \\
\hline
\end{tabular}

Table 12. Quimichule canyon.

\begin{tabular}{|c|c|c|c|c|c|c|c|}
\hline \multicolumn{8}{|c|}{ Strength calculation of water } \\
\hline Pto. & Altitude & $\begin{array}{l}\text { Density of } \\
\text { water }\end{array}$ & $m=\frac{N_{2}-N_{1}}{W_{2}-W_{1}}$ & $\begin{array}{l}\text { Cosine of the } \\
\text { slope (Cos m) }\end{array}$ & $\begin{array}{c}\text { Acceleration } \\
\text { gravity local (gl) }\end{array}$ & Partial forces & $F_{i}=\left(\mathrm{d}^{*} \cos m\right)(g l)$ \\
\hline \multicolumn{8}{|c|}{ Channel slope } \\
\hline 1 & 3920 & & & & & & \multirow[b]{2}{*}{$\sum F_{i}=1173.08566882$} \\
\hline 2 & 3680 & 1000 & -0.416168 & 0.999974 & 9.774448 & 97.741897 & \\
\hline 3 & 3500 & 1000 & -0.414141 & 0.999974 & 9.775002 & 97.747464 & \\
\hline 4 & 3360 & 1000 & 0.398168 & 0.999976 & 9.775434 & 97.751975 & \multirow{2}{*}{ Data } \\
\hline 5 & 3250 & 1000 & -0.114741 & 0.999998 & 9.775773 & 97.757530 & \\
\hline 6 & 3150 & 1000 & -0.417382 & 0.999973 & 9.776081 & 97.768215 & \multirow{3}{*}{12} \\
\hline 7 & 3080 & 1000 & -0.412557 & 0.999974 & 9.776297 & 97.760431 & \\
\hline 8 & 3030 & 1000 & -0.000042 & 1.000000 & 9.776451 & 97.764509 & \\
\hline 9 & 2990 & 1000 & 0.000046 & 1.000000 & 9.776574 & 97.765740 & \multirow{3}{*}{$F=\sum_{i=1}^{n} F_{i}$} \\
\hline 10 & 2900 & 1000 & -0.348323 & 0.999982 & 9.776850 & 97.766690 & \\
\hline 11 & 2840 & 1000 & -0.417100 & 0.999974 & 9.777033 & 97.767740 & \\
\hline 12 & 2780 & 1000 & 0.582900 & 0.999948 & 9.777216 & 97.767101 & \multirow[b]{2}{*}{$97.75713907 \mathrm{~N}$} \\
\hline 13 & 2730 & 1000 & 1.582900 & 0.999618 & 9.777369 & 97.736376 & \\
\hline
\end{tabular}


Table 13. Colorada canyon.

\begin{tabular}{ccc}
\hline \multicolumn{3}{c}{ Calculation of the waste } \\
\hline Area of drainage basin & 2835645.45 & $\mathrm{~m}^{2}$ \\
Channel length & 5500 & $\mathrm{~m}$ \\
Permeability coefficient $(c)$ & 0.05 & $\mathrm{~cm} / \mathrm{s}$ \\
Rainfall rate $(i)$ & 0.000000 & $\mathrm{~m}$ \\
Waste & 0.000000 & $\mathrm{~m}^{3} / \mathrm{s}$ \\
\hline
\end{tabular}

Table 14. Quimichule canyon.
\begin{tabular}{ccc}
\hline \multicolumn{3}{c|}{ Calculation of the waste } \\
\hline Area of drainage basin & 2990700 & $\mathrm{~m}^{2}$ \\
Channel length & 6500 & $\mathrm{~m}$ \\
Permeability coefficient (c) & 0.05 & $\mathrm{~cm} / \mathrm{s}$ \\
Rainfall rate $(i)$ & 0.000000 & $\mathrm{~m}$ \\
Waste & 0.000000 & $\mathrm{~m}^{3} / \mathrm{s}$ \\
\hline
\end{tabular}

where:

$Q=$ Waste in $\mathrm{m}^{3} / \mathrm{s}$

$k=$ permeability coefficient

$i=$ Hydraulic gradient

$A=$ Capture area

$0.278=$ Conversion factor

\section{Conclusions}

First of all, it is worth mentioning that no similar analysis has been found within the literature related with research studies on the Popocatépetl volcano. This paper presents the results obtained through the processing of the cartographic data and by applying the Geoeas and Surfer programs to calculate the relation between the acceleration of local gravity, height, slope, waste and force of a substance that runs over a profile of the Colorada and Quimichule canyons.

Gravity, slope, force and waste are lower in the Colorada canyon, results validating the methods applied. The correlation coefficient between gravity and height shows that there is a perfect negative correlation, i.e. the higher the height, the lower the gravity.

It is given that the absolute value of the flow rate obtained in each canyon is not comparable, since the surface of the Barranca Colorada is only 54.5\% of the Barranca Quimichule, and to compare it is necessary to obtain the specific flow of each (dividing its surface, which is usually given in $\mathrm{m}^{3} / \mathrm{s}^{*} \mathrm{~km}^{2}$ or $1 / \mathrm{s}^{*} \mathrm{~km}^{2}$ ).

The specific flows of each canyon would then be:

Colorada $\left(68.528454 \mathrm{~m}^{3} / 2.3563 \mathrm{~s} \mathrm{~km}^{2}\right)=0.2908311 \mathrm{~m}^{3} / \mathrm{s} \mathrm{km}^{2}$ or $290.8311 \mathrm{l} / \mathrm{s} \mathrm{km}^{2}$

Quimichule $\left(110.050759 \mathrm{~m}^{3} / 4.324587 \mathrm{~s} \mathrm{~km}^{2}\right)=0.254477 \mathrm{~m}^{3} / \mathrm{s} \mathrm{km}^{2}$ or $254.477 \mathrm{l} / \mathrm{s} \mathrm{km}^{2}$

Therefore, Quimichule canyon produces per $\mathrm{km}^{2} 87.5 \%$ of water that runs off the Colorada canyon, the latter being the one that would be able to produce a greater runoff. This result can be compared to the acceleration of local gravity of $9.7760 \mathrm{~m} / \mathrm{s}^{2}$ in Quimichule and $9.7753 \mathrm{~m} / \mathrm{s}^{2}$ in Colorada with the values of force of water of 97.76131777 $\mathrm{N}$ in Quimichule and 97.75423653 $\mathrm{N}$ in Colorado, with the average values of the slope of -0.166153846 in Quimichule and -0.196923077 in Colorada, revealing that the few differences of local gravity $(0.0007 \%$ greater in Quimichule) have an equivalent influence over the force of water, which suggests that this is due primarily to differences in altitude that show both canyons.

However, these variables do not intervene in the calculation of the flows (Q) where the difference of the average slope or hydraulic gradient (i) determines the theoretical calculating of the flows. This same difference 
observed between the values of (i) is present in the values of $(\mathrm{Q})$ (87.5\% between Quimichule and Colorada).

The application of geostatistical models highlights the importance of applying mathematics in geomorphological analysis, presenting different graphs and comparative data analysis and structural modeling studies in geomorphological and hydrological processes that are useful to get an idea of the behavior of the water flow, mud or magma, caused by rain or activity of Popocatépetl, which could affect the communities near the canyons.

This study aims to contribute to the existing work, and its results obtained by using technological tools applied in the analysis are considered as a valuable contribution to the natural disaster prevention field. We put special emphasis on the fact that the application of different models would certainly lead to similar results.

\section{References}

[1] García, F., Ramos, R. and Domínguez, R. (1996) Posible flujo de lodo en el costado oriente del volcán Popocatépetl. En el libro Volcán Popocatépetl, Estudios Realizados Durante la Crisis de 1994-1995. Sistema Nacional de Protección Civil, Centro Nacional de Prevención de Desastres, Universidad Nacional Autónoma de México, 109-125.

[2] Franco, J.M., Cassano, A.M. and Bolla, G.L. (2005) Estabilidad de barrancas sobre el río Paraná. Departamento de Ingeniería Civil de la Universidad Regional de Paraná. Universidad Tecnológica Nacional, 1-119.

[3] Spiegel, M. (2000) Probabilidad y Estadística. Mc. Graw Hill, México.

[4] Ramos, R., Máximo, P., Narciso, J., Mirón, M. and Beltrán, M. (2012) Estudio geoestadístico para obtener la gravedad local, pendiente y cálculo hidrológico de las barrancas Xaltelulco, Tepeloncocone y Tenepanco del volcán Popocatépetl. Boletín de Ciencias de la Tierra, 31, 65-84.

[5] Ramos, R., Máximo, P., Montiel, A., González, Y. and Rodríguez, A. (2010) Análisis fotogramétrico del volcán Citlaltépetl. Cartográfica, 84, 105-116.

[6] Gasquet, C. (2000) Analyse de fourier et applications. Dunod, France.

[7] Godman, P. (2001) Principles of Geographical Information Systems for Land Resources. Springer, USA.

[8] Schenk, T. (2002) Fotogrametría digital. Marcombo-ICC, España.

[9] Lennon, T. (2002) Remote Sensing Digital Image Analysis. Esa/Esrin, USA. 